\title{
Peripheral and Autonomic Neuropathy in South Asians and White Caucasians with Type 2 Diabetes Mellitus: Possible Explanations for Epidemiological Differences
}

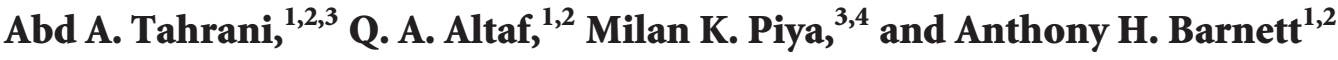 \\ ${ }^{1}$ Institute of Metabolism and Systems Research, University of Birmingham, Birmingham, UK \\ ${ }^{2}$ Department of Diabetes and Endocrinology, Heart of England NHS Foundation Trust, Birmingham, UK \\ ${ }^{3}$ Centre of Endocrinology, Diabetes and Metabolism, Birmingham Health Partners, Birmingham, UK \\ ${ }^{4}$ Department of Diabetes and Endocrinology, Derby Teaching Hospitals NHS Foundation Trust, Derby, UK
}

Correspondence should be addressed to Abd A. Tahrani; abd.tahrani@nhs.net

Received 21 November 2016; Revised 1 February 2017; Accepted 19 February 2017; Published 20 March 2017

Academic Editor: Patrizio Tatti

Copyright (C) 2017 Abd A. Tahrani et al. This is an open access article distributed under the Creative Commons Attribution License, which permits unrestricted use, distribution, and reproduction in any medium, provided the original work is properly cited.

\begin{abstract}
Objectives. To compare the prevalence of diabetic peripheral neuropathy (DPN) and that of cardiac autonomic neuropathy (CAN) between South Asians and White Caucasians with type 2 diabetes and to explore reasons for observed differences. Methods. A cross-sectional study of casually selected South Asian and White Caucasian adults attending a hospital-based diabetes clinic in the UK. DPN and CAN were assessed using the Michigan Neuropathy Screening Instrument (MNSI) and heart rate variability testing, respectively. Results. Patients $(n=266)$ were recruited ( $47.4 \%$ South Asians). DPN was more common in White Caucasians compared to South Asians (54.3\% versus 38.1\%, $p=0.008$ ). Foot insensitivity as assessed by $10 \mathrm{~g}$ monofilament perception was more common in White Caucasians (43.9\% versus $23.8 \%, p=0.001)$. After adjustment for confounders, White Caucasians remained twice as likely to have DPN as South Asians, but the impact of ethnicity became nonsignificant after adjusting for adiposity measures or height. No difference in prevalence of standardized CAN test abnormalities was detected between ethnicities. Skin microvascular assessment demonstrated that South Asians had reduced heating flux but preserved acetylcholine response. Conclusions. South Asians with type 2 diabetes have fewer clinical signs of DPN compared to White Caucasians. Differences in adiposity (and its distribution) and height appear to explain these differences.
\end{abstract}

\section{Introduction}

Diabetic neuropathy (DN) is the most common form of neuropathy in the Western world and one of the most troublesome complications of diabetes mellitus, resulting in great morbidity (e.g., it is the leading cause of nontraumatic lower limb amputation), mortality, and significant economic burden [1-3]. The reported prevalence of diabetic peripheral neuropathy (DPN) and diabetic autonomic neuropathy (DAN) varies significantly depending on the population studied and the methods used [2-9]. Many factors have been implicated in the pathogenesis of $\mathrm{DN}$ including nonmetabolic factors such as age and height $[3,6,10,11]$, as well as metabolic factors such as hyperglycemia, hypertension, dyslipidemia, waist circumference, and obesity [3, 12-14].
These metabolic factors stimulate multiple pathways resulting in direct cellular damage and functional and/or structural defects involving the extracellular matrix and/or microvasculature $[15,16]$. Disease-modifying treatments are still lacking (with the exception of improved glycaemic control) [12, 15]. Hence, improved understanding of DN pathogenesis is important in order to identify new treatment targets [15].

South Asians with type 2 diabetes seem to be at lower risk of developing DPN resulting in a lower incidence of foot ulcerations and amputations [17-19]. Such a difference is surprising as diabetes-related complications have predominantly a vascular aetiology, with South Asian patients at higher risk of cardiovascular disease compared to White Caucasians with type 2 diabetes [20]. However, the previous reports that identified this difference either conducted a very 
limited assessment of DPN $[17,20]$ or utilized a patient population with a low prevalence of diabetes complications and could not detect a difference in DPN prevalence using routine clinical examination techniques [18]. Critically, the underlying factors that could explain such differences in DPN prevalence have not been fully explored. One previous report, based on a primary care population in the UK, suggested that differences in height and transcutaneous partial pressure of oxygen (TCpO2) were responsible for the lower prevalence of DPN in South Asians [18]. This report, however, did not adjust for a wide range of possible confounders such as obesity, alcohol intake, and triglycerides (amongst others), all of which are well established risk factors for neuropathy.

In contrast to DPN, the impact of ethnicity on CAN in patients with type 2 diabetes has received little attention, with the exception of a single report that compared the $E / I$ ratios between South Asians and White Caucasians [18]; hence there is need for further studies utilizing more sensitive and detailed assessments of CAN.

The primary aim of this study was therefore to explore the possible metabolic, demographic, and microvascular differences that might explain the lower prevalence of DPN in South Asians with type 2 diabetes compared to White Caucasians. Secondary aims included comparing the prevalence of clinically detectable DPN and that of cardiac autonomic neuropathy (CAN) in South Asians and White Caucasians with type 2 diabetes receiving hospital-based diabetes care.

\section{Materials and Methods}

This is a secondary analysis of an ongoing project with the aim of exploring the mechanisms contributing to the development of microvascular complications in patients with type 2 diabetes [21-24]. We have conducted a cross-sectional study of casually selected South Asian or White Caucasian adults with type 2 diabetes recruited from the clinic of a hospital-based diabetes centre in the UK. Subjects excluded were those under the age of 18, not of either South Asian or White Caucasian ethnicity, or known to have neuropathy for other reasons apart from diabetes $(<1 \%)$.

Patients were recruited casually from the outpatient diabetes departments of two different hospitals in the UK. The majority of participants (86\%) were recruited from the Heart of England NHS Foundation Trust in Birmingham, UK, which has a large South Asian population with type 2 diabetes (approximately $40 \%$ of the total clinic). These factors suggest that our sample is representative and the two ethnicities are comparable. Patients who originated from India, Pakistan, Sri Lanka, or Bangladesh were considered to be of South Asian origin. Ethnicity was determined using the UK decennial census by the study participants. The project was approved by the Warwickshire Research Ethics Committee (REC number 08/H1211/145). Patients were consented prior to participation in the study.

DPN was assessed using the Michigan Neuropathy Screening Instrument (MNSI) which is a validated, 2-component tool designed to facilitate the early diagnosis of DPN [4, 25-29]. The questionnaire component (MNSIq) comprises 15 questions seeking to characterize sensory disturbance but also peripheral vascular disease and general asthenia [25]. The examination component (MNSIe) comprises a limited foot inspection to identify deformity, skin abnormalities, and ulceration, coupled with an assessment of the vibratory perception and ankle tendon reflexes [25]. For the purpose of this study, DPN was diagnosed if the MNSIe score was $>2$ and/or MNSIq was $\geq 7$ [27]. We have also used perception to a $10 \mathrm{~g}$ monofilament (applied to 10 positions, the tip of each toe, under 3 metatarsal heads, the plantar surface of the foot, and the dorsal space between the first and second toe) as a test for foot insensitivity; an abnormal monofilament test was defined as $<8$ correct responses [30]. The relationship of ethnicity to neuropathic symptoms was also assessed using the MNSIq.

CAN was assessed using heart rate variability (HRV) and was analyzed using the continuous wavelet transform methods to generate numerical and graphical data using the ANX3.0 software, ANSAR Inc., Philadelphia, PA. The R-R intervals were recorded and the HRV was plotted in the frequency domain to separate the high-frequency (Rfa, 0.15 to $0.4 \mathrm{~Hz}$ ) from the low-frequency ( $\mathrm{Lfa}, 0.04$ to $0.15 \mathrm{~Hz}$ ) components by spectral analysis. HRV and BP were recorded with the patient in sitting position during resting, deep breathing, Valsalva maneuver, and standing position [31]. Data recorded included the $E / I$ (expiratory/inspiratory) ratio, Valsalva ratio, $30: 15$ ratio, frequency domain analysis with respiratory adjustment (adjusted low frequency (Lfa), adjusted respiratory frequency (Rfa), and Lfa/Rfa), and time domain analysis including standard deviation of beat-to-beat intervals ( $\mathrm{dNN})$, root mean square of successive differences (rmsSD), and the proportion of NN50 (pNN50). For the purpose of this study, a diagnosis of CAN was made when 2 or more of the following tests were abnormal: $E / I$ ratio, Valsalva ratio, $30: 15$ ratio, and postural drop in $\mathrm{BP}$ (drop of $20 \mathrm{mmHg}$ in systolic or $10 \mathrm{mmHg}$ in diastolic BP) [2]. Age-related normal values for $E / I$, Valsalva, and $30: 15$ ratios were defined as previously reported [32].

Microvascular and endothelial assessment were performed on a casually chosen subset of patients $(n=71)$ using Laser speckle contrast imaging (Moor Instruments Ltd., Devon, UK) which has been shown to be reproducible [33]. All patients were approached to have the vascular/endothelial assessment and the test was performed on all those who agreed. The South Asians and White Caucasians in this subset had similar characteristics to those in the total study population reported in Table 1. Microvascular blood flow (measures in arbitrary perfusion units (APU)) was assessed at the left mid-thigh level. This site was chosen to minimise the impact of any peripheral vascular disease in the lower limbs. Measurements were taken with the patient in a semirecumbent position in a room temperature of $22-23^{\circ} \mathrm{C}$ following 15 minutes of patients acclimatisation [34]. Imaging was performed over 20 minutes, and all measurements were taken simultaneously. Blood flow was measured at baseline (for 20 minutes) and following maximal dilatation following heating to $44^{\circ} \mathrm{C}$. Endothelial function was measured following the iontophoresis of $1 \%$ acetylcholine (Ach) and 2\% sodium nitroprusside (SNP) (5 pulses over 5 minutes). Data are presented as absolute values, conductance (measured as flux 
TABLE 1: Summary of baseline characteristics in relation to ethnicity. Data are presented as median (IQR) or mean $\pm 1 S D$ depending on data distribution. The percentages represent $\%$ of participants in the ethnic group. STR: sight threatening retinopathy defined as preproliferative or proliferative retinopathy or maculopathy or previous laser treatment. TIA: transient ischaemic attack. PVD: peripheral vascular disease.

\begin{tabular}{|c|c|c|c|}
\hline & $\begin{array}{l}\text { South Asians } \\
(n=126)\end{array}$ & $\begin{array}{l}\text { White Caucasians } \\
\quad(n=140)\end{array}$ & $p$ value \\
\hline Age (years) & $54.9 \pm 12.5$ & $59.2 \pm 10.8$ & 0.003 \\
\hline Male $(\%)$ & 60.3 & 56.4 & 0.521 \\
\hline Smoking (current or ex-smoker) & 30.2 & 50.7 & 0.001 \\
\hline Alcohol (\%) & 2.4 & 47.9 & $<0.001$ \\
\hline Diabetes duration (years) & $11.0(7.0-18.0)$ & $10.5(5.0-16.0)$ & 0.153 \\
\hline $\operatorname{BMI}\left(\mathrm{kg} / \mathrm{m}^{2}\right)$ & $30.1(26.5-33.6)$ & $35.3(31.8-41.4)$ & $<0.001$ \\
\hline Waist circumference $(\mathrm{cm})$ & $103.2(96.9-113.1)$ & $117.2(109.2-129.4)$ & $<0.001$ \\
\hline Hip circumference $(\mathrm{cm})$ & $102.5(97-112.25)$ & $120.0(108.6-131.75)$ & $<0.001$ \\
\hline Waist/hip ratio & $1.00 \pm 0.06$ & $0.99 \pm 0.11$ & 0.276 \\
\hline Neck circumference $(\mathrm{cm})$ & $39.0(36.9-41.8)$ & $43.0(39.6-47.0)$ & $<0.001$ \\
\hline Height $(\mathrm{cm})$ & $164.5 \pm 8.9$ & $167.1 \pm 12.3$ & 0.051 \\
\hline Neck height ratio & $0.24 \pm 0.02$ & $0.26 \pm 0.03$ & $<0.001$ \\
\hline Systolic blood pressure (mmHg) & $126.0(115.5-138.5)$ & $130.0(124.5-140.0)$ & 0.008 \\
\hline Diastolic blood pressure (mmHg) & $77.5(70.0-84.5)$ & $79.0(73.6-85.9)$ & 0.032 \\
\hline HbAlc (\%) & $8.0(7.16-9.20)$ & $8.0(7.23-9.19)$ & 0.868 \\
\hline Total cholesterol (mmol/L) & $3.7(3.3-4.3)$ & $3.7(3.3-4.4)$ & 0.637 \\
\hline Triglycerides (mmol/L) & $1.8(1.1-2.4)$ & $1.7(1.3-2.5)$ & 0.550 \\
\hline $\mathrm{HDL}(\mathrm{mmol} / \mathrm{L})$ & $1.1(0.9-1.2)$ & $1.1(0.9-1.3)$ & 0.217 \\
\hline $\mathrm{TSH}(\mathrm{mU} / \mathrm{L})$ & $1.6(1.0-2.3)$ & $1.9(1.4-2.3)$ & 0.024 \\
\hline Estimated GFR $\left(\mathrm{mL} / \mathrm{min} / 1.73 \mathrm{~m}^{2}\right)$ & $90.0 \pm 25.5$ & $83.7 \pm 27.1$ & 0.053 \\
\hline Oral antidiabetes agent (\%) & 91.3 & 92.9 & 0.632 \\
\hline Sulphonylureas (\%) & 42.1 & 30.7 & 0.054 \\
\hline Insulin (\%) & 50.0 & 57.9 & 0.199 \\
\hline Insulin dose (units) & $80.0(44.0-118)$ & $67.0(52.0-97.5)$ & 0.649 \\
\hline GLP-1 analogues $(\%)$ & 4.0 & 17.1 & 0.001 \\
\hline Lipid lowering treatment (\%) & 81 & 85 & 0.379 \\
\hline Antihypertensive therapy & 74.6 & 84.3 & 0.05 \\
\hline Antiplatelet agents (\%) & 65.6 & 64.5 & 0.847 \\
\hline Albuminuria (\%) & 38.6 & 34.6 & 0.536 \\
\hline $\operatorname{STR}(\%)$ & 36.0 & 36.8 & 0.908 \\
\hline Ischaemic heart disease (\%) & 23.0 & 17.1 & 0.231 \\
\hline CABG $(\%)$ & 13.5 & 10.0 & 0.375 \\
\hline Stroke/ TIA (\%) & 9.5 & 10.7 & 0.748 \\
\hline $\operatorname{PVD}(\%)$ & 2.4 & 8.6 & 0.029 \\
\hline
\end{tabular}

divided by mean arterial pressure in order to account for differences in BP) and as percentage of maximal dilatation flow as recommended by previous reports [34]. Heating response represented maximum dilatation [34].

Data analysis was performed using SPSS 15.0 software (SPSS Inc., Chicago, USA). Data are presented as mean \pm 1 SD or median (IQR) depending on data distribution. Normality testing was performed using histograms and the ShapiroWilk test. Independent continuous variables were compared using the independent $t$-test or the Mann-Whitney test. Categorical variables were compared using the Chi square test. The Bonferroni correction was applied to define statistical significance when the components of the MNSIe and MNSIq were compared between the ethnicities. In order to identify independent predictors of DPN and CAN (separately), logistic regression models were applied. Variables included in logistic regression models were tested for multicollinearity using the tolerance test and the variance inflation factor; no evidence of multicollinearity was found in the logistic regression models used in this paper. Adiposity measures (BMI, waist, and neck circumferences) were included in the regression models separately due to the high correlation between these variables. A $p$ value $<0.05$ was considered significant throughout the manuscript except when stated otherwise. 


\section{Results}

Patients $(n=266)$ were included in this analysis, $47.4 \%$ of whom were South Asian. South Asians were shorter and younger but had similar duration of known diabetes. South Asians also had lower adiposity measurements, BP, TSH levels, smoking, and alcohol intake (Table 1). The prescription of antihypertensive and GLP-1 analogue treatment was also lower in South Asians (Table 1). The prevalence of other microvascular complications and past medical history of coronary artery disease was similar between ethnicities, while South Asians had lower prevalence of peripheral vascular disease (PVD) (Table 1) which is consistent with other previous reports [17].

DPN was more common in White Caucasians compared to South Asians (54.3\% versus 38.1\%, $p=0.008$ ). Foot insensitivity, as assessed by abnormal monofilament perception, was also more common in White Caucasians (43.9\% versus $23.8 \%, p=0.001$ ) (Table 2). Analysis of patient symptom scores demonstrated that symptoms consistent with sensory deficit were not different between ethnic groups whereas pain/discomfort symptoms related to were nonsignificantly more common in South Asians (Table 2). White Caucasian patients had more abnormalities on all aspects of neuropathy examination and, consistent with our findings with the monofilament, reported more open sores on the foot (Table 2).

To determine the potential influence of demographic and metabolic differences between ethnicities on the prevalence of DPN, logistic regression models with increasing complexity were used (Table 3 ). The association between ethnicity and DPN remained significant, despite adjusting for a wide range of possible confounders. This association was attenuated by age and a history of PVD and was abolished after adjusting for height and adiposity measures (with the exception of waist/hip ratio) (Table 3 ), suggesting that ethnic differences in DPN prevalence can be mainly explained by the differences in height and adiposity between the ethnic groups. All adiposity measures were independently associated with DPN despite adjusting for all possible confounders listed in Table 3 (except height and BMI which were not used in the same model) [BMI: odds ratio (OR) 1.076 (95\% CI 1.026 to 1.130$), p=$ 0.003; waist circumference: OR 1.040 (95\% CI 1.016 to 1.064), $p=0.001$; neck circumference: OR 1.143 (95\% CI 1.045 to $1.250), p=0.003$ ]. Other independent associations included previously reported measures such as age, diabetes duration, and insulin treatment $[13,14,35]$.

Unlike clinically apparent DPN, we found no difference in prevalence of abnormalities of standardized cardiovascular autonomic function tests between ethnicities (40.9\% versus $40.9 \%, p=0.995)$. Frequency and time domain analysis, however, revealed more extensive deficits in the White Caucasian compared to the South Asian cohort (Table 4). However, these differences were not significant after adjusting for possible confounders (such as age). Diabetes duration [OR 1.084 (95\% CI 1.028 to 1.144 ), $p=0.003$ ] and the use of calcium channel blockers [OR 0.292 (95\% CI 0.123 to 0.694 ), $p=0.005$ ] were found to be independently associated with CAN after adjusting the data, but not ethnicity [OR 1.077 (95\% CI 0.473 to 2.452), $p=0.860$ ].
Microvascular and endothelial assessment demonstrated significant differences between the two ethnicities. South Asians had similar baseline skin flux and conductance and markedly lower maximal dilatation following heating despite a similar SNP response but had preserved endothelialdependant vasodilatation (Table 5). Indeed, endothelialdependent vasodilatation significantly exceeded that measured in the White Caucasian population when expressed as the ratio of Ach-induced to heating-induced flux [34] (Table 5).

\section{Discussion}

We have compared the prevalence of DPN and that of CAN between South Asians and White Caucasians with type 2 diabetes from a hospital-based care setting. We then tried to determine whether specific metabolic and/or demographic factors could explain observed differences in the prevalence of clinically detectable DPN and CAN between these groups. Our study clearly demonstrates that DPN and foot insensitivity are less common in South Asian patients and implicates height and adiposity as potential factors underlying this difference. Furthermore, we found that total body (BMI), visceral (waist circumference), and upper body (neck circumference) fat were independently associated with DPN after adjusting for a range of confounders.

Our diabetes centre serves an area that has a high proportion of South Asians, although the majority of the general population are White Caucasians (a reflection of the much higher prevalence of type 2 diabetes in South Asians in the UK and elsewhere). Both ethnicities live in the same geographical area, have similar deprivation scores, and are referred to our clinic by primary care physicians using the same referral guidelines which are preagreed with our centre. Furthermore, there are no differences in clinic attendance rates between the ethnicities at our centre. Moreover, our population of South Asians and White Europeans with type 2 diabetes has similar characteristics (such as age, gender, smoking, alcohol, BMI, and HbAlc) to those described in previous reports in primary care in other localities in the UK. As our study population was hospital-based and with consequently more advanced disease, our findings are not necessarily generalizable to the general population of patients with type 2 diabetes (please see below) [18].

Our data demonstrating a lower prevalence of DPN in South Asian subjects are consistent with other reports [1719] but extend these findings by demonstrating significant differences detectable by routine clinical examination and the novel finding that obesity (and its distribution) explains, at least in part, the ethnic differences in DPN prevalence. In addition, previous study populations exploring the relationships of DPN and ethnicity were drawn from Primary Care Practices in the UK which was reflected in the shorter duration of diabetes, the lower prevalence of microvascular, and macrovascular complications and the less frequent use of insulin and lipid lowering treatments $[18,19]$, while our study extends the findings to hospital-based population. Our study patients were drawn from a population receiving hospitalbased diabetes care and are therefore representative of a more 


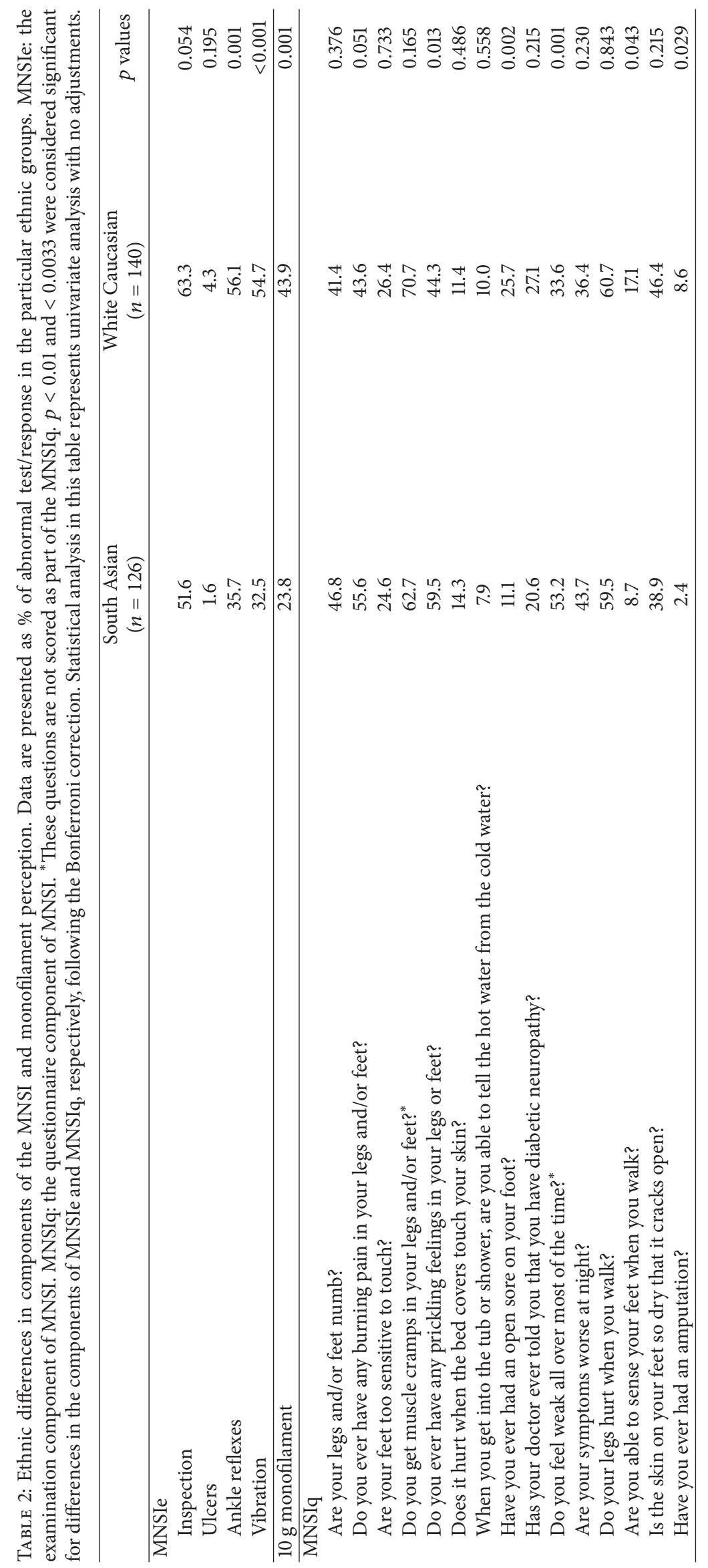


TABLE 3: Assessing the impact of possible confounders on the association between ethnicity and DPN (based on MNSI) using logistic regression models with increasing complexity. The odds ratios reported are the odds for having DPN in White Caucasians to South Asians. BP: blood pressure; eGFR: estimated glomerular filtration rate; PVD: peripheral vascular disease; BMI: body mass index.

\begin{tabular}{|c|c|c|c|c|}
\hline Model & Nagelkerke $R^{2}$ & Odds ratio & $\begin{array}{c}95 \% \text { confidence } \\
\text { interval }\end{array}$ & $p$ value \\
\hline Unadjusted: ethnicity & 0.035 & 1.930 & $1.182-3.149$ & 0.009 \\
\hline Model 1: ethnicity + age + gender & 0.091 & 1.684 & $1.016-2.793$ & 0.043 \\
\hline 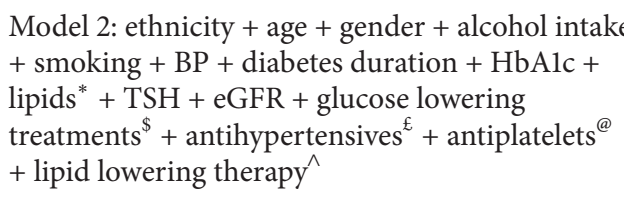 & 0.224 & 1.987 & $1.069-3.693$ & 0.030 \\
\hline Model 3: Model 2 + PVD & 0.237 & 1.887 & $1.008-3.530$ & 0.047 \\
\hline Model 4: Model 3 + height & 0.244 & 1.766 & $0.932-3.348$ & 0.081 \\
\hline Model 5: Model 3 + BMI & 0.279 & 1.169 & $0.581-2.352$ & 0.661 \\
\hline Model 6: Model 3 + waist circumference & 0.290 & 1.077 & $0.532-2.180$ & 0.837 \\
\hline Model 7: Model 3 + neck circumference & 0.278 & 1.087 & $0.528-2.237$ & 0.822 \\
\hline
\end{tabular}

${ }^{*}$ Adjustment for lipids included adjustment for total cholesterol, triglycerides, and HDL individually.

\$Adjustment for glucose lowering treatments included adjustment for metformin, sulphonylurea, glitazones, DPP-4 inhibitors, insulin, and GLP-1 analogues individually. No other glucose lowering treatment was used in our study population.

${ }^{E}$ Adjustment for antihypertensives included adjustment for ACE inhibitors, angiotensin 2 blockers, beta blockers, alpha blockers, calcium antagonists, and diuretics individually.

${ }^{@}$ Antiplatelets included aspirin and clopidogrel.

$\wedge^{\wedge}$ Adjustment for lipid lowering therapy included adjustment for statins, ezetimibe, and fibrates individually.

TABLE 4: Differences in heart rate variability, spectral analysis, and time-domain and frequency-domain parameters between South Asians and White Caucasians with diabetes. Nonsignificant comparisons from the HRV and frequency and time domain analysis have not been included. Data are presented as median (IQR).

\begin{tabular}{lccc}
\hline & $\begin{array}{c}\text { South Asians } \\
(n=115)\end{array}$ & $\begin{array}{c}\text { White } \\
\text { Caucasians } \\
(n=110)\end{array}$ & $p$ value \\
\hline $30: 15$ ratio & $1.26(1.11-1.60)$ & $1.19(1.08-1.37)$ & 0.027 \\
Baseline Lfa & $0.95(0.42-2.14)$ & $0.60(0.27-1.38)$ & 0.041 \\
Baseline Rfa & $0.54(0.21-1.19)$ & $0.35(0.15-0.80)$ & 0.032 \\
Deep breathing Lfa & $0.70(0.29-1.84)$ & $0.44(0.16-1.35)$ & 0.035 \\
Standing Lfa & $1.13(0.41-2.73)$ & $0.69(0.23-1.43)$ & 0.018 \\
\hline
\end{tabular}

advanced, complicated disease, which has not previously been assessed. This difference in population characteristics may underlie the previously reported inability to detect significant differences in DPN prevalence using the Neuropathy Disability Score (NDS), instead relying upon findings from nerve electrophysiology [18]. Moreover, although not directly assessed, preservation of the microcirculation was implicated as a potential contributor to the lower risk of DPN and foot ulceration observed in South Asian patients [18]. There was, however, no adjustment performed for adiposity.

Our study confirms previous reports that foot ulceration is lower in South Asian compared to White Caucasian patients with type 2 diabetes [17]. This finding is consistent with our observation of relative preservation of $10 \mathrm{~g}$ monofilament perception in South Asian patients. Interestingly, the overall prevalence of neuropathic symptoms ascribable to
TABLE 5: Assessment of microvascular blood flow and endothelial function in South Asians and White Caucasians with type 2 diabetes. Data presented as median (IQR) or ratios. Blood flux was measured in arbitrary perfusion units (APU). Conductance is the measure of dividing flux by the mean arterial pressure.

\begin{tabular}{lccc}
\hline & $\begin{array}{c}\text { South Asians } \\
(n=33)\end{array}$ & $\begin{array}{c}\text { White Caucasians } \\
(n=38)\end{array}$ & $\begin{array}{c}p \text { value, } \\
\text { unadjusted }\end{array}$ \\
\hline Flux \\
Baseline & $23.10(17.30-35.15)$ & $24.20(15.70-35.22)$ & 0.986 \\
Heating & 140.10 & 174.00 \\
(107.30-169.05) & $(143.12-207.62)$ & 0.006 \\
Ach & 112.00 & $107.40(73.62-142.77)$ & 0.991 \\
SNP & $(84.80-135.50)$ & \\
\hline \multicolumn{5}{c}{ Conductance } \\
Baseline & $0.27(0.18-0.40)$ & $0.27(0.17-0.37)$ & 0.653 \\
Heating & $1.58(1.22-1.90)$ & $1.77(1.50-2.19)$ & 0.029 \\
Ach & $1.26(0.88-1.38)$ & $1.07(0.77-1.53)$ \\
SNP & $1.12(0.75-1.51)$ & $1.30(0.88-1.84)$ & 0.612 \\
\hline \multicolumn{5}{c}{ Flux in relation to maximum vasodilatation } \\
Baseline & $0.17(0.12-0.24)$ & $0.15(0.10-0.21)$ \\
Ach & $0.78(0.62-0.91)$ & $0.63(0.43-0.76)$ \\
SNP & $0.78(0.46-1.01)$ & $0.75(0.51-0.94)$ & 0.074 \\
\hline
\end{tabular}

DPN, however, was comparable across ethnicities with similar percentages reporting symptoms consistent with sensory loss but (nonsignificantly) more South Asian patients reporting neuropathic pain. It is known that South Asians have a higher prevalence of vitamin D deficiency [36], which might 
contribute to differences in some symptoms, particularly generalized weakness.

Our results showed that age, diabetes duration, and insulin treatment were independently associated with DPN, as has been previously reported $[13,14,35]$. The relationship between DPN and insulin is interesting, but due to the crosssectional nature of this study, causation cannot be proven. Intensive insulin treatment by improving metabolic control has been shown to have beneficial effects on DPN [12]. Patients might have been started on insulin because of the presence of DPN and other microvascular complications in order to slow progression. On the other hand, our data do not show a relationship between DPN and other risk factors such as triglycerides [37]; this could be related to our sample size, the different characteristics of our study population, or the high proportion of patients that were on lipid lowering therapy.

South Asians have different body fat distribution to White Europeans and tend to have greater fat mass than White Europeans for the same BMI level [38-40]. In order to address this issue, we used different measures of adiposity including BMI, waist circumference, and neck circumference, all of which were greater in White Europeans compared to South Asians in our study. All of these adiposity measures removed the impact of ethnicity on DPN when added to the regression model.

Our data, demonstrating BMI, waist circumference, and neck circumference as independent predictors of DPN, is potentially of mechanistic importance. Neck circumference is associated with cardiometabolic risk factors [41] and with obstructive sleep apnoea (OSA) [42], a disease that is very common in type 2 diabetes (up to $86 \%$ prevalence) [43] and is known to be associated with endothelial dysfunction and abnormal blood flow regulation [44]. Interestingly, neck circumference has recently been identified as an independent predictor of diabetic retinopathy [45].

Maximal skin blood flow of the lower limb on heating was reduced but endothelial function preserved in South Asian compared to White Caucasians with type 2 diabetes. In contrast, there were no significant differences in the response to SNP. To our knowledge, this is the first evaluation of skin microvascular flow regulation in South Asian patients with diabetes. Heating to $44^{\circ} \mathrm{C}$ leads to maximal vasodilatation which corresponds to the maximal vasodilatory capacity [34]. Deficits in heating responses have been associated with increased prevalence of cardiovascular disease $[46,47]$ and so our findings might have relevance for the higher cardiovascular disease risk observed in South Asians with type 2 diabetes [20].

In contrast to the heating response, the response to Ach (when measured as a ratio of maximal vasodilatation) was greater in South Asians, suggesting relative preservation of endothelial-dependent vasodilatation. The response to Ach is thought to reflect both an axon reflex and prostaglandinmediated component [34] which might, therefore, be of relevance to the lower prevalence of DPN and foot complications in these subjects. In patients without diabetes, heating response was not different [47] while SNP was lower [48] in South Asians compared to White Caucasians when measured in the forearm. Our data are also consistent with the finding of relative preservation of $\mathrm{TCpO} 2$ in South Asian subjects with diabetes [18].

Unlike DPN, there was no difference in the prevalence of abnormalities of standardized CAN tests between South Asian and White Caucasian patients, although differences in spectral analysis suggested more preserved autonomic function in South Asians. This highlights the fact that the pathogenesis of DPN and CAN may not be the same and understanding such differences will be important to develop targeted treatments. Differences in CAN prevalence between South Asian and White Caucasian patients with type 2 diabetes have been previously assessed using the $E / I$ ratio and the postural drop in BP as markers of CAN [18]. In this previous report, there was no difference in the postural drop between ethnicities (consistent with our findings), but there was a significant difference in the change in heart rate associated with deep breathing between ethnicities. It is difficult to directly compare our results with the results from this report, as we performed a more extensive assessment of CAN and we have presented our $E / I$ ratio data as categorical variable rather than an absolute change in heart rate.

There are some limitations of our study, particularly the differences in baseline parameters such as age, height, BP, smoking, and alcohol consumption between the ethnic groups which might have affected the associations observed in our study. However, the principal findings of our study remained significant after adjusting for a wide range of possible confounders. Matching ethnicities for some of the baseline parameters might have been preferred, but such precise matching is difficult (particularly regarding age and adiposity measures) due to the significant demographic differences that exist between our local South Asian and White Caucasian population with type 2 diabetes. The relatively small size of our sample might have obscured the relationship between CAN and ethnicity. Nonetheless, this sample size was adequate to detect a difference in the prevalence of DPN consistent with the construct that differences in the pathogenesis of peripheral and autonomic neuropathy may exist. In addition, this is a cross-sectional study and hence causation cannot be proven. Additionally, there is the possibility of self-selection bias (i.e., patients with microvascular complications preferentially agreeing to participate), which cannot be entirely excluded. However, all patients attending a general diabetes clinic were approached about willingness to enrol in the study and we are unaware of an ethnicity-mediated self-selection bias which would affect our conclusions. This is further supported by the similar prevalence of diabetic retinopathy between the two ethnicities. Finally, the MNSI is not the "gold standard" for diagnosing DPN but it has been validated against nerve conduction studies $[25,29]$ and has been used widely in landmark studies $[4,26,27,30]$. We chose to use the MNSI (in concert with the $10 \mathrm{~g}$ monofilament) since it offers the advantage of providing robust, meaningful, clinically detectable end-points.

In summary, clinical signs consistent with DPN are reduced in South Asian compared to White Caucasian patients with type 2 diabetes receiving hospital-based diabetes care. In contrast, the overall prevalence of neuropathic 
symptoms ascribable to DPN was similar in these ethnic groups. Differences in height and adiposity appear to explain the ethnic differences in DPN prevalence; and obesity was an independent determent of DPN in these populations. We have also identified a novel association between neck circumference and DPN which might be of pathogenic importance as neck circumference is associated with cardiometabolic risk factors and OSA. Endothelial-dependent microvascular function, but not the response to heating, was also relatively preserved in South Asian subjects. Future studies are warranted to characterize small nerve fibre loss and dysfunction in different populations, address the possible role of OSA in the pathogenesis of DPN, and further explore differences in microvascular blood flow regulation between different ethnicities.

\section{Abbreviations}

DN: Diabetic neuropathy

DPN: Diabetic peripheral neuropathy

DAN: Diabetic autonomic neuropathy

CAN: Cardiac autonomic neuropathy

TCpO2: Transcutaneous partial pressure of oxygen

MNSI: Michigan Neuropathy Screening Instrument

HRV: Heart rate variability

VLF: Very low frequency

LF: $\quad$ Low frequency

HF: $\quad$ High frequency

NDS: Neuropathy Disability Score

AGE: Advanced glycation end-product.

\section{Disclosure}

Dr. Abd Tahrani is a NIHR Clinician Scientist. The views expressed in this publication are those of the authors and not necessarily those of the NHS, the National Institute for Health Research, or the Department of Health.

\section{Conflicts of Interest}

The authors declare that they have no conflicts of interest.

\section{Authors' Contributions}

Abd A. Tahrani contributed to conception, design, analysis, interpretation, writing first draft, and final approval. Q. A. Altaf contributed to reviewing draft and final approval. Milan K. Piya contributed to conduct of the study, data collection, reviewing the draft, and final approval. Anthony $\mathrm{H}$. Barnett contributed to design, reviewing draft, and final approval.

\section{Acknowledgments}

Dr. Abd Tahrani is a Clinician Scientist supported by the National Institute for Health Research. The authors acknowledge Dr. Fahmy Hanna, Mrs. Helen Hodgson, and Mrs. Rebecca Barakam from the University Hospital of North Staffordshire for their help in recruitment.

\section{References}

[1] A. I. Vinik, T. S. Park, K. B. Stansberry, and G. L. Pittenger, “Diabetic neuropathies," Diabetologia, vol. 43, no. 8, pp. 957973, 2000.

[2] A. I. Vinik and D. Ziegler, "Diabetic cardiovascular autonomic neuropathy," Circulation, vol. 115, no. 3, pp. 387-397, 2007.

[3] D. Ziegler, K. Dannehl, H. Muhlen, M. Spuler, and F. A. Gries, "Prevalence of cardiovascular autonomic dysfunction assessed by spectral analysis, vector analysis, and standard tests of heart rate variation and blood pressure responses at various stages of diabetic neuropathy," Diabetic Medicine, vol. 9, no. 9, pp. 806814, 1992.

[4] The DCCT Research Group, "Factors in development of diabetic neuropathy. Baseline analysis of neuropathy in feasibility phase of Diabetes Control and Complications Trial (DCCT)," Diabetes, vol. 37, no. 4, pp. 476-481, 1988.

[5] M. Toeller, A. E. Buyken, G. Heitkamp, G. Berg, and W. A. Scherbaum, "Prevalence of chronic complications, metabolic control and nutritional intake in type 1 diabetes: comparison between different European regions," Hormone and Metabolic Research, vol. 31, no. 12, pp. 680-685, 1999.

[6] R. E. Maser, A. R. Steenkiste, J. S. Dorman et al., "Epidemiological correlates of diabetic neuropathy. Report from Pittsburgh epidemiology of diabetes complications study," Diabetes, vol. 38, no. 11, pp. 1456-1461, 1989.

[7] C. N. De Wytt, R. V. Jackson, G. I. Hockings, J. M. Joyner, and C. R. Strakosch, "Polyneuropathy in Australian outpatients with type II diabetes mellitus," Journal of Diabetes and Its Complications, vol. 13, no. 2, pp. 74-78, 1999.

[8] M. J. Stevens, F. Dayanikli, D. M. Raffel et al., "Scintigraphic assessment of regionalized defects in myocardial sympathetic innervation and blood flow regulation in diabetic patients with autonomic neuropathy," Journal of the American College of Cardiology, vol. 31, no. 7, pp. 1575-1584, 1998.

[9] M. J. Stevens, D. M. Raffel, K. C. Allman et al., "Cardiac sympathetic dysinnervation in diabetes: implications for enhanced cardiovascular risk," Circulation, vol. 98, no. 10, pp. 961-968, 1998.

[10] J. Partanen, L. Niskanen, J. Lehtinen, E. Mervaala, O. Siitonen, and M. Uusitupa, "Natural history of peripheral neuropathy in patients with non-insulin- dependent diabetes mellitus," New England Journal of Medicine, vol. 333, no. 2, pp. 89-94, 1995.

[11] J. P. Töyry, L. K. Niskanen, M. J. Mäntysaari, E. A. Länsimies, and M. I. J. Uusitupa, "Occurrence, predictors, and clinical significance of autonomic neuropathy in NIDDM: ten-year followup from the diagnosis," Diabetes, vol. 45, no. 3, pp. 308-315, 1996.

[12] The Diabetes Control and Complications Trial Research Group, "The effect of intensive treatment of diabetes on the development and progression of long-term complications in insulindependent diabetes mellitus," The New England Journal of Medicine, vol. 329, no. 14, pp. 977-986, 1993.

[13] S. Tesfaye, N. Chaturvedi, S. E. M. Eaton et al., "Vascular risk factors and diabetic neuropathy," New England Journal of Medicine, vol. 352, no. 4, pp. 341-431, 2005.

[14] J. Elliott, S. Tesfaye, N. Chaturvedi et al., "Large-fiber dysfunction in diabetic peripheral neuropathy is predicted by cardiovascular risk factors," Diabetes Care, vol. 32, no. 10, pp. 18961900, 2009. 
[15] A. A. Tahrani, T. Askwith, and M. J. Stevens, "Emerging drugs for diabetic neuropathy," Expert Opinion on Emerging Drugs, vol. 15, no. 4, pp. 661-683, 2010.

[16] M. Brownlee, "The pathobiology of diabetic complications," Diabetes, vol. 54, no. 6, pp. 1615-1625, 2005.

[17] C. A. Abbott, A. P. Garrow, A. L. Carrington, J. Morris, E. R. Van Ross, and A. J. Boulton, "Foot ulcer risk is lower in SouthAsian and African-Caribbean compared with European diabetic patients in the U.K.," Diabetes Care, vol. 28, no. 8, pp. 1869$1875,2005$.

[18] C. A. Abbott, N. Chaturvedi, R. A. Malik et al., "Explanations for the lower rates of diabetic neuropathy in Indian Asians versus Europeans," Diabetes Care, vol. 33, no. 6, pp. 1325-1330, 2010.

[19] N. Chaturvedi, C. A. Abbott, A. Whalley, P. Widdows, S. Y. Leggetter, and A. J. M. Boulton, "Risk of diabetes-related amputation in South Asians vs. Europeans in the UK," Diabetic Medicine, vol. 19, no. 2, pp. 99-104, 2002.

[20] A. H. Barnett, A. N. Dixon, S. Bellary, M. W. Hanif, J. P. O’Hare, and N. T. Raymond, "Type 2 diabetes and cardiovascular risk in the UK south Asian community," Diabetologia, vol. 49, no. 10, pp. 2234-2246, 2006.

[21] A. A. Tahrani, A. Ali, N. T. Raymond et al., "Obstructive sleep apnea and diabetic neuropathy: a novel association in patients with type 2 diabetes," American Journal of Respiratory and Critical Care Medicine, vol. 186, no. 5, pp. 434-441, 2012.

[22] A. A. Tahrani, A. Ali, N. T. Raymond et al., "Obstructive sleep apnea and diabetic nephropathy: a cohort study," Diabetes Care, vol. 36, no. 11, pp. 3718-3725, 2013.

[23] A. A. Tahrani, K. Dubb, N. T. Raymond et al., "Cardiac autonomic neuropathy predicts renal function decline in patients with type 2 diabetes: a cohort study," Diabetologia, vol. 57 , no. 6 , pp. 1249-1256, 2014.

[24] A. A. M. Tahrani, Microvascular complications in patients with type 2 diabetes: the impact of ethnicity, sleep and oxidative stress [Ph.D. thesis], University of Birmingham, 2013.

[25] E. L. Feldman, M. J. Stevens, P. K. Thomas, M. B. Brown, N. Canal, and D. A. Greene, "A practical two-step quantitative clinical and electrophysiological assessment for the diagnosis and staging of diabetic neuropathy," Diabetes Care, vol. 17, no. 11, pp. 1281-1289, 1994.

[26] Epidemiology of Diabetes Interventions and Complications (EDIC), "Design, implementation, and preliminary results of a long-term follow-up of the Diabetes Control and Complications Trial cohort," Diabetes Care, vol. 22, no. 1, pp. 99-111, 1999.

[27] C. L. Martin, J. Albers, W. H. Herman et al., "Neuropathy among the diabetes control and complications trial cohort 8 years after trial completion," Diabetes Care, vol. 29, no. 2, pp. 340-344, 2006.

[28] O. Boyraz and M. Saracoglu, "The effect of obesity on the assessment of diabetic peripheral neuropathy: a comparison of Michigan patient version test and Michigan physical assessment," Diabetes Research and Clinical Practice, vol. 90, no. 3, pp. 256260, 2010.

[29] A. Moghtaderi, A. Bakhshipour, and H. Rashidi, "Validation of Michigan neuropathy screening instrument for diabetic peripheral neuropathy," Clinical Neurology and Neurosurgery, vol. 108, no. 5, pp. 477-481, 2006.

[30] G. Pambianco, T. Costacou, E. Strotmeyer, and T. J. Orchard, "The assessment of clinical distal symmetric polyneuropathy in type 1 diabetes: a comparison of methodologies from the Pittsburgh Epidemiology of Diabetes Complications Cohort,"
Diabetes Research and Clinical Practice, vol. 92, no. 2, pp. 280287, 2011.

[31] J. P. Colombo, W. C. M. Shoemaker, H. M. Belzberg, G. M. Hatzakis, P. M. Fathizadeh, and D. M. Demetriades, "Noninvasive monitoring of the autonomic nervous system and hemodynamics of patients with blunt and penetrating trauma," Journal of Trauma-Injury, Infection \& Critical Care, vol. 65, no. 6, pp. 1364-1373, 2008.

[32] D. Ziegler, G. Laux, K. Dannehl et al., "Assessment of cardiovascular autonomic function: age-related normal ranges and reproducibility of spectral analysis, vector analysis, and standard tests of heart rate variation and blood pressure responses," Diabetic Medicine, vol. 9, no. 2, pp. 166-175, 1992.

[33] M. Roustit, C. Millet, S. Blaise, B. Dufournet, and J. L. Cracowski, "Excellent reproducibility of laser speckle contrast imaging to assess skin microvascular reactivity," Microvascular Research, vol. 80, no. 3, pp. 505-511, 2010.

[34] J.-L. Cracowski, C. T. Minson, M. Salvat-Melis, and J. R. Halliwill, "Methodological issues in the assessment of skin microvascular endothelial function in humans," Trends in Pharmacological Sciences, vol. 27, no. 9, pp. 503-508, 2006.

[35] R. Pop-Busui, J. Lu, N. Lopes, and T. L. Z. Jones, "Prevalence of diabetic peripheral neuropathy and relation to glycemic control therapies at baseline in the BARI 2D cohort," Journal of the Peripheral Nervous System, vol. 14, no. 1, pp. 1-13, 2009.

[36] A. A. Tahrani, A. Ball, L. Shepherd, A. Rahim, A. F. Jones, and A. Bates, "The prevalence of vitamin D abnormalities in South Asians with type 2 diabetes mellitus in the UK," International Journal of Clinical Practice, vol. 64, no. 3, pp. 351-355, 2010.

[37] T. D. Wiggin, K. A. Sullivan, R. Pop-Busui, A. Amato, A. A. F. Sima, and E. L. Feldman, "Elevated triglycerides correlate with progression of diabetic neuropathy," Diabetes, vol. 58, no. 7, pp. 1634-1640, 2009.

[38] A. H. Barnett, A. N. Dixon, S. Bellary et al., "Type 2 diabetes and cardiovascular risk in the UK south Asian community," Diabetologia, vol. 49, no. 10, pp. 2234-2246, 2006.

[39] L. J. Gray, T. Yates, M. J. Davies et al., "Defining obesity cutoff points for migrant South Asians," PLoS ONE, vol. 6, no. 10, Article ID e26464, 2011.

[40] F. Razak, S. S. Anand, H. Shannon et al., "Defining obesity cut points in a multiethnic population," Circulation, vol. 115, no. 16, pp. 2111-2118, 2007.

[41] S. R. Preis, J. M. Massaro, U. Hoffmann et al., "Neck circumference as a novel measure of cardiometabolic risk: the framingham heart study," Journal of Clinical Endocrinology and Metabolism, vol. 95, no. 8, pp. 3701-3710, 2010.

[42] B. Caffo, M. Diener-West, N. M. Punjabi, and J. Samet, "A novel approach to prediction of mild obstructive sleep disordered breathing in a population-based sample: the sleep heart health study," Sleep, vol. 33, no. 12, pp. 1641-1648, 2010.

[43] G. D. Foster, M. H. Sanders, R. Millman et al., "Obstructive sleep apnea among obese patients with type 2 diabetes," Diabetes Care, vol. 32, no. 6, pp. 1017-1019, 2009.

[44] S. Yim-Yeh, S. Rahangdale, A. T. D. Nguyen et al., "Vascular dysfunction in obstructive sleep apnea and type 2 diabetes mellitus," Obesity, vol. 19, no. 1, pp. 17-22, 2011.

[45] M. Dirani, J. Xie, E. Fenwick et al., "Are obesity and anthropometry risk factors for diabetic retinopathy?: the diabetes management project," Investigative Ophthalmology and Visual Science, vol. 52, no. 7, pp. 4416-4421, 2011. 
[46] P. Dandona, H. Ghanim, A. Chaudhuri, and P. Mohanty, “Thiazolidinediones-improving endothelial function and potential long-term benefits on cardiovascular disease in subjects with type 2 diabetes," Journal of Diabetes and Its Complications, vol. 22, no. 1, pp. 62-75, 2008.

[47] W. D. Strain, A. D. Hughes, J. Mayet et al., "Attenuation of microvascular function in those with cardiovascular disease is similar in patients of Indian Asian and European descent," BMC Cardiovascular Disorders, vol. 10, no. 1, article 3, 2010.

[48] A. Misra and L. Khurana, "Obesity-related non-communicable diseases: South Asians vs White Caucasians," International Journal of Obesity, vol. 35, no. 2, pp. 167-187, 2011. 


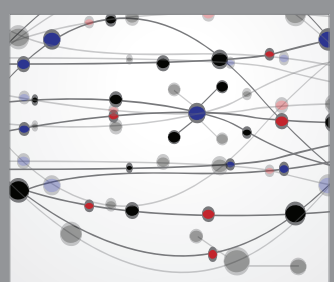

The Scientific World Journal
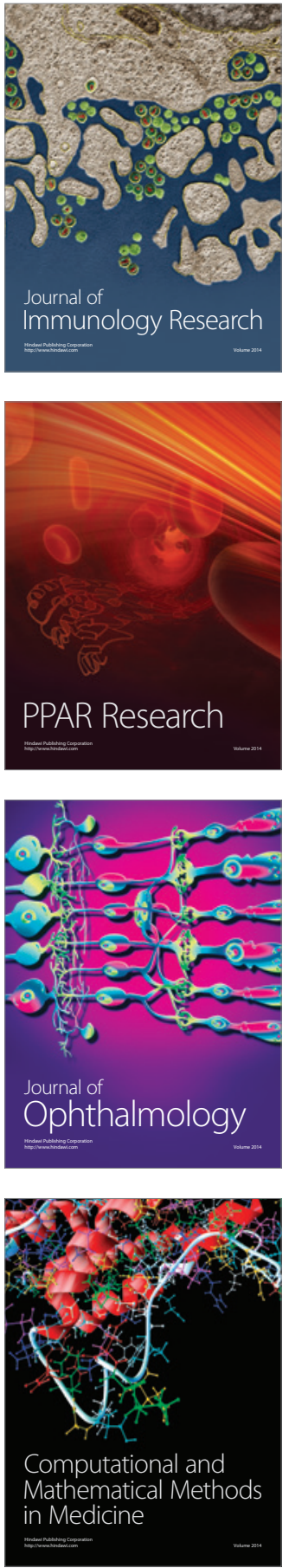

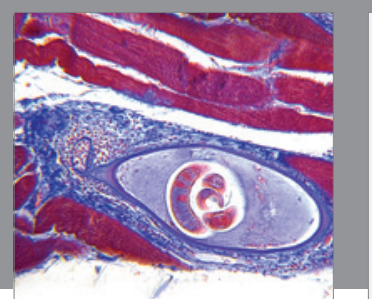

Gastroenterology Research and Practice
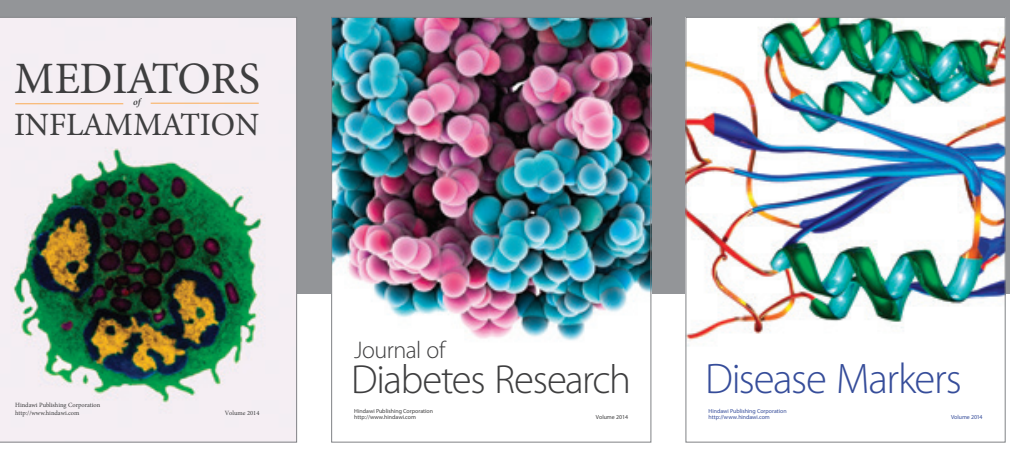

Disease Markers

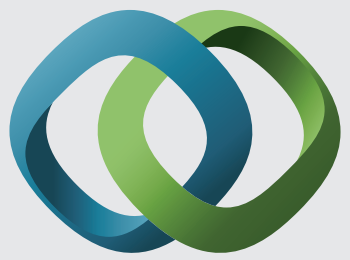

\section{Hindawi}

Submit your manuscripts at

https://www.hindawi.com
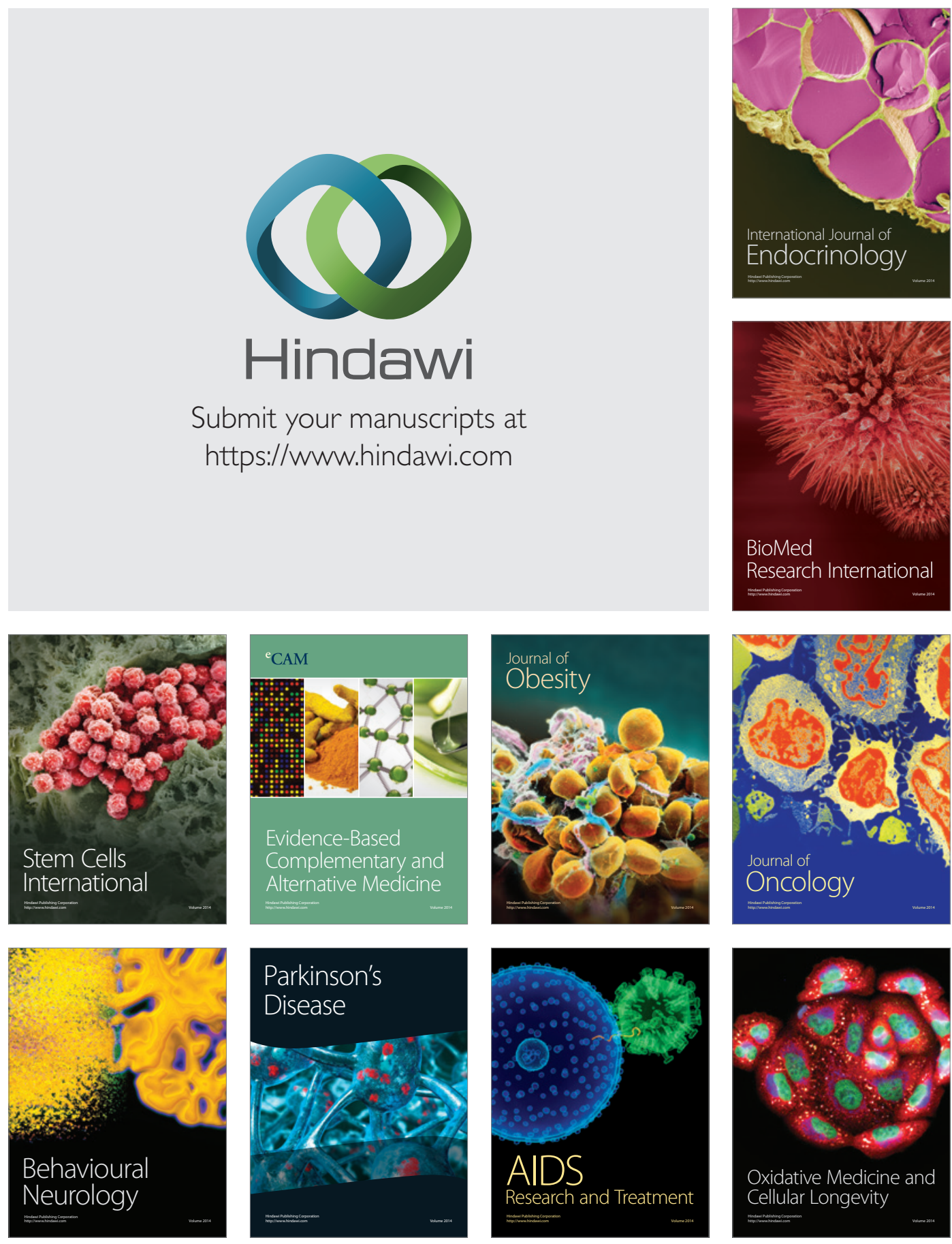